\title{
INFLUENCES OF CHLORPROMAZINE ON ALLERGIC RESPONSE OF RABBIT SPLEEN, A HISTOLOGICAL STUDY
}

\author{
KYUHEI NAKADATE*, NIRO ISONO, \\ SHIGEAKI KAMIKATAHIRA and YUICHI ABE \\ Department of Legal Medicine, School of Medicine, \\ Keio University
}

(Received on September 11, 1957)

\section{INTRODUCTION}

Nowadays, it is well known that Chlorpromazine (CP for short in the followings) is used, in an extensive range, not only clinically for artificial hibernation or for narcosis-intensifying remedy, but for cramp-stopping, as a sedative or analgetic and furthermore for treatment of various kinds of psychosis, and that its effects have been demonstrated.

The chief functions of $\mathrm{CP}$ are undoubtedly related to the autonomous or central nervous system. A plenty of experiments have already explained that it restrains strikingly the functions of the autonomous nervous system and reduced or decreased the various reactions and metabolism in organisms ${ }^{(1 \sim 3)}$. Although now about the pharmacological functions of $\mathrm{CP}$ a good deal of interesting facts have been found one by one ${ }^{(4 \sim 5)}$, many subjects of the functional mechanism of $\mathrm{CP}$ as an antidote to shock, have not entirely been made clear. Particularly fundamental experimental studies on the influenses to shock are unexpectedly in a small number.

Recently, Nakayama ${ }^{(6)}$ confirmed that $\mathrm{CP}$ has a remarkable restraining function to passive anaphylactic shock and a protective ability to Arthus' phenomenon in rabbits. The authors, for the purpose of confirming the influences of $\mathrm{CP}$ to allergic response in visceral organs, provoked experimentally tissue allergy and observed histologically the changes caused by $\mathrm{CP}$, and obtained following new findings.

\section{MATERIALS AND METHODS}

Healthy male rabbits, weighing about $2.0 \mathrm{~kg}$, of same age, were used in the experiments. Besides the control group of four animals, they were divided

\footnotetext{
* Professor of Legal Medicine.
} 
into the following ten groups (four rabbits in each). Except the sensitized group, all the animals were slaughtered by air embolism, after 24 hours from the last procedure. Here, as CP the 0.5 percent Contomin solution offered by Yoshitomi Co. was used.

(1) Group of sensitation: Horse serum $1.0 \mathrm{ml} / \mathrm{kg}$ was injected seven times alternately into the auricular vein and the peritoneal cavity, once a day. The animals were killed after six days from the last injection.

(2) Group of tissue allergy: After seven days from the above mentioned sensitation, horse serum was injected into the splenic artery of laparotomized animals. The abdominal walls were immediately closed after the injections.

(3) Group of preliminary CP treatment: After seven days from the last sensitation, $0.2 \mathrm{mg} / \mathrm{kg}$ CP was intravenously injected five times every one hour, and furthermore after one hour $0.2 \mathrm{ml}$ horse serum was injected into the splenic artery.

(4) Group of simultaneous CP treatment: At the same time of the injections for tissue allergy as in the Group 2, $0.2 \mathrm{mg} \mathrm{CP}$ was injected into the splenic artery.

(5) Group of later CP treatment: After one hour from the injection like as in the group $2,0.2 \mathrm{mg} / \mathrm{kg} \mathrm{CP}$ was injected intravenously and afterwards the same dose of the drug, four times every one hour, was injected into the auricular vein.

(6) Group of preliminary treatment with saline solution: After seven days from the last sensitation, $5.0 \mathrm{ml}$ of physiological saline solution was injected intravenously, and one hour after this procedure, $0.2 \mathrm{ml}$ horse serum was injected into the splenic artery.

(7) Group of simultaneous treatment with saline solution: At the same time of allergy provocation, physiological saline solution was injected into the splenic artery.

(8) Group of later treatment with saline solution: After one hour from the allergy provocation, $5.0 \mathrm{ml}$ physiological saline solution was injected into the auricular vein.

(9) Group of CP injection: Instead of the allergy provoking injection, $0.2 \mathrm{mg}$ CP was injected into the splenic artery.

(10) Group of saline injection: Instead of the allergy provoking injection, physiological saline solution was injected into the splenic artery.

The spleens, removed after the last procedure, were fixed in ten per cent formalin solution, in absolute alcohol or in Helly's solution. Then, the paraffin sections were made and stained with hematoxylin-eosin, methylgreen pyronine, 
thionine (differentiated with ten per cent perchloric acid), Feulgen-Rossenbeck's staining and Gomori's periodic acid methenamin silver method (modified by Jones and Yajima).

Stillmore, after six days from the sensitation and just before the slaughter, venous blood was taken and the precipitin titre was estimated. It was convinced that the titre amounted to 5,000 to $7,000 \times$ in the group of sensitation, to 6,000 to $8,000 \times$ in the group 1 , to 5,000 to $8,000 \times$ in the group 2 , to 5,000 to $7,000 \times$ in the group 3 , to 5,000 to $8,000 \times$ in the group 4 , to 6,000 to $9,000 \times$ in the group 5 , to 5,000 to $8,000 \times$ in the group 6 , to 5,000 to $8,000 \times$ in the group 7 , to 4,000 to $6,000 \times$ in the group 8 and to 5,000 to $7,000 \times$ in the group 9 .

\section{RESULTS}

(1) Controls.-The capsule and trabeculae were smooth. The follicles were roundish or elliptic and their boundaries were generally distinct. In the follicles a plenty of small lymphocytes, of which the nuclear structure was indistinct and greater parts of nuclei were without cell plasm, or of medium large lymphocytes of which chromatin nets were rough, were closely gathered. A small number of large lymphocytes, of which cytoplasm was easily stained by pyronine, were observed here and there. So called typical large plasmacells of "Malshalko" type with eccentric and spoke like nuclei, perinuclear pale area and strikingly basophile cytoplasm, appeared in a small number. Germinal centers in the follicles were not numerously found. The central arteries were vacant and no degenerative change was observed in the walls of them. The red pulp was generally scanty of blood. The reticular cells were ordinary arranged and the reticular fibers were well preserved. Endothel cells of the venous sini had no swelling and peeling off, and the structures were well maintained. The cells, in which ribonucleic acid (RNA) and desoxyribonucleic acid (DNA) were positive, were seen in the white pulp in a large number, but scattered in the red pulp.

(2) Group of sensitation.-The capsule and trabeculae were slightly thickened, and proliferation of young connective tissue was observed. White pulp was distinct on its boundary and enlarged. The germinal centers in the nodules appeared abundantly, and the proliferation of lymphoblasts or large lymphocytes, of which cytoplasm was light colored and nuclei were swollen, were demonstrated. Thickening and marked swelling of the cells of the central artery walls were found. In the red pulp, various grades of congestion existed and slight effusion of blood plasm were observed. Swelling and increase of reticular cells and proliferation of reticular fiber net works were brought about. Furthermore, the lining cells of the venous sini were swollen and RNA positive 
cells were increased more markedly than in the control group.

(3) Group of tissue allergy.-Capsule and trabeculae were swollen, and infiltration of young fibroblasts was found. White pulp was enlarged and its boundaries were somewhat indistinct. The networks of reticular fibers in the follicles proliferated considerably and separation was observed. In the follicles lymphoblasts or large lymphocytes with swollen nuclei crowded together, but cells with distinctly structured nuclei were rather in a small number, and various grades of degeneration of nucleus, such as edema, vacuolation, karyorrhexis, pyknosis, karyolysis were demonstrated. Germinal center formation in the nodules were slightly seen. The reticular cells were increased, but they showed gerenally cloudy swelling of some intensive grades. And especially in the surroundings of the centers in the nodules, typical plasmacells proliferated and infiltrated. The central artery walls were thickened and the fibrinoid degeneration was brought on, and the cells of wall had tendency to cloudy swelling. In the red pulp, congestion and hemorrhage of medium grade existed and erythroclasis was remarkable. Plasmic effusion was seen here. There was deposit of hemosiderin and phagocytosis was remarkably found with increase of monocytes. The lining cells of the venous sini swelled and their turbidity and falling off were seen. The networks of reticular fibers hypertrophied and proliferated generally, but their arrangement was irregularly separated. In the splenic cords, infiltration of polymorphonuclear leucocytes was observed. The DNA positivity had no marked change, and the RNA positivity was indefinite, because the same findings were seen even in the cells with destroyed nuclei.

(4) Group of preliminary CP treatment.--The capsule was thickened in some parts and was smooth in some parts. Marked swelling and proliferation of the connective tissue were brought about. The trabeculae had the similar findings. The lymphfollicles were roundish or elliptic, and the appearance of the germinal centers was marked in general. In the centers of the nodules, typical reticular cells appeared and light sighted, large round cells proliferated strikingly. The nuclei of these cells were large and light sighted, and the chromatin nets were subtle and the nucleic membranes were clear. Giant nucleoli, which were roundish or polymorphous, existed in the center of nuclei or near the nucleic membrane. The number of nucleoli was one or several. The large, round cytoplasm and giant nucleoli were diffusely stained with pyronine and the karyoplasm was slightly stained with methylgreen. The color of pyronine of the plasm disappeared by differentiation. It seemed that these cells were so called "lymphogonia-Amano" advocated by Amano, Unno and others(12 14). In the surroundings of the germinal centers, small or medium sized lymphocytes with masses of thick 
chromatin crowded together. The central artery walls were thickened and the swollen wall cells were found, but fibrinoid or hyaline degeneration were almost undemonstrable. In the red pulp, congestion of medium grade was obsereved and hemosiderin deposit was considerably seen. Swollen and increased reticular cells or erythrophagia by monocytes were found. The lining cells of venous sini were remarkably swollen but no evidence of their falling off was seen. RNA positivity was highest in this group and it was markedly demonstrated especially in the lymphoblasts of the white pulp. DNA positivity was also increased.

(5) Group of simultaneous CP treatment.- The capsule and trabeculae were slightly thickend. Lymph follicles were enlarged but the germinal centers were not observable. Lymphocyte arrangement in the white pulp was relatively close. Increase of lymphoblasts and large lymphocytes was seen here and most of them ware stained with alkaline pigments homogenously and intensively. The nucleic membranes were indistinct. In the splenic cords around lymph follicles, intensive hemorrhage and congestion were often brought about. Erythrocytes united each other and became heteromorphic with findings of blood plasma effusion. The reticular cells became turbid and reticular fiber networks atrophied. The reticular fiber arrangement was somewhat irregularly separated. RNA positive cells were rather markedly weakened in stainability.

(6) Group of later CP treatment.-Findings of this group were almost similar to the group of preliminary CP treatment. But arrangement of lymphoblasts in the white pulp was rather scattered and at the same time destruction of lymphocytes was found. Beside proliferation of splenic cells, degenerative changes of nuclei, such as karyorrhexis and vacuolation, and increase of polymorphonuclear leukocytes were observed. In the red pulp, congestion of medium degree, remarkable destruction of erythrocytes and marked deposit of hemcsiderin were brought on in place to place.

(7) Group of preliminary treatment with saline solution.-All the findings of this group resembled to the above mentioned group of allergy. Namely, the capsule and trabeculae were swollen and the cells in the white pulp proliferated, but the degenerations, like opaque karyoplasm, deformity of nuclei and turbidity of nucleic membrane, were observed. The central artery walls thickened and their fibrinoid degeneration was seen. In the surroundings of the germinal centers, proliferation and infiltration of typical plasma cells were demonstrated. Proliferation and turbidity of splenic cells, hemosiderin deposition and erythrophagia were all remarkable. The structure of venous sini fell into disorder, and with cloudy swelling or falling off of the lining cells, increase of polymorphonuclear leukocytes was observed. Change of nucleic acid positivity almost agreed 
Table 1

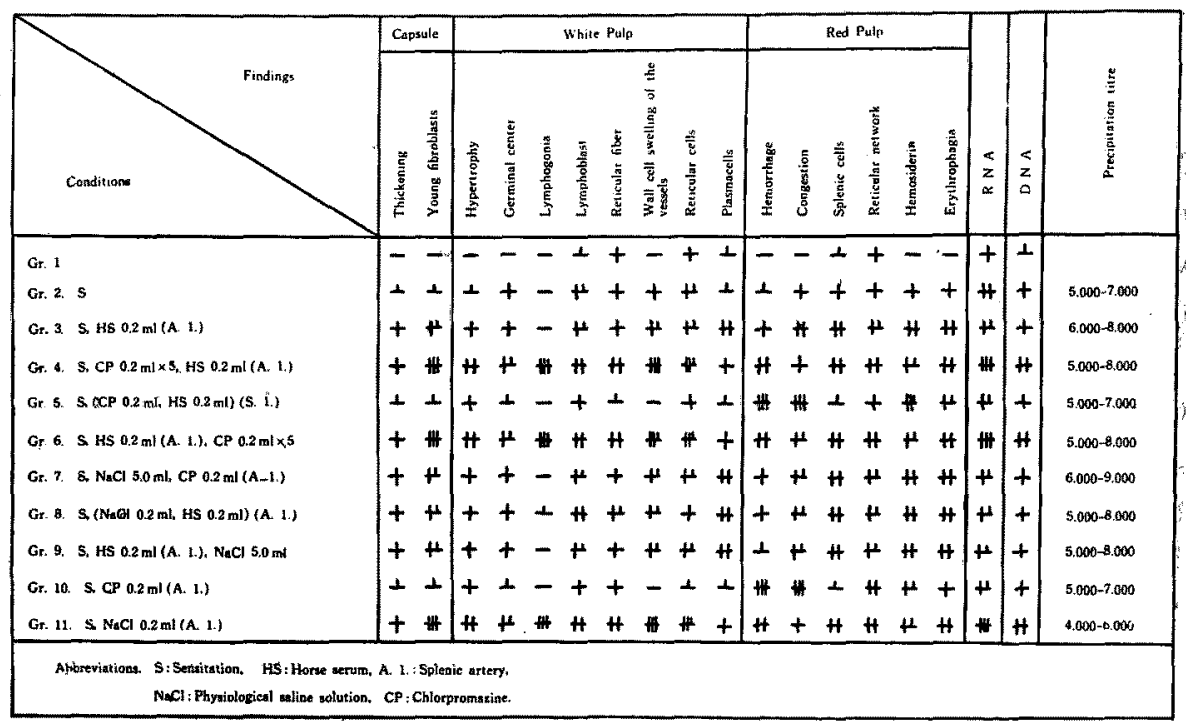

with that of the group of tissue allergy.

(8) Group of simultaneous treatment with saline solution.-The outlineof findings resembled to it of the group of preliminary treatment with saline solution. But in the white pulp, large leukocytes with distinct nucleic structure often appeared, and degeneration of the splenic cells and congestion were ratherslight.

(9) Group of later treatment with saline solution.-The findings were similar to those of the group of preliminary treatment with saline solution, and no particular difference was found.

(10) Group of CP injection.-The findings of this group were almost similar to the group of simultaneous CP treatment.

(11) Group of saline injection.-In this group the appearance of a large number of lymphoblasts in the white pulp was characteristic, and the findings resembled to those of the group of preliminary $\mathrm{CP}$ treatment. But the swelling and increase of the lining cells of venous sini and the proliferation of the splenic cells were all in slight degree (See the table 1).

\section{DISCUSSION}

On the bases of the above mentioned findings, the authors showed thefollowing matters.

1. Groups of sensitation: Enlargement of the lymph follicles, germinal. 
center formation, appearance of young lymphocytes, proliferation and swelling of the reticular cells and of network of reticular fibers and congestion were observed and the reaction of cellular proliferation and infiltration were demonstrated.

2. Groups of tissue allergy, of preliminary, simultaneous and later treatment with saline solution: Swelling of the connective tissue, proliferation, infiltration destruction and degeneration of young lymphocytes and reticular cells, cloudy swelling and falling off of lining cells of the sini, appearance of polymorphonuclear leukocytes and of typical plasma cells, and congestion were found. Cellular proliferative and infiltrative reaction, serous infiltration and dissolution reaction of the tissue, degeneration and necrosis displayed states of confusion.

3. Groups of preliminary and later CP treatment and of saline injection: In the lymph follicles, marked proliferation and infiltration, something like the lymphoblasts with abundance of RNA, and swelling and proliferation of the reticular systems were characteristic. It was considered that the facts belonged to the extreme types of cellular proliferative and infiltrative reactions.

4. Groups of simultaneous CP treatment and of CP injection: Close crowd and degeneration of young lymphocytes and reticular cells, atrophy of the reticular network fibers, congestion and hemorrhage were present. It could be asserted that the facts were the characteristic findings of cellular proliferative

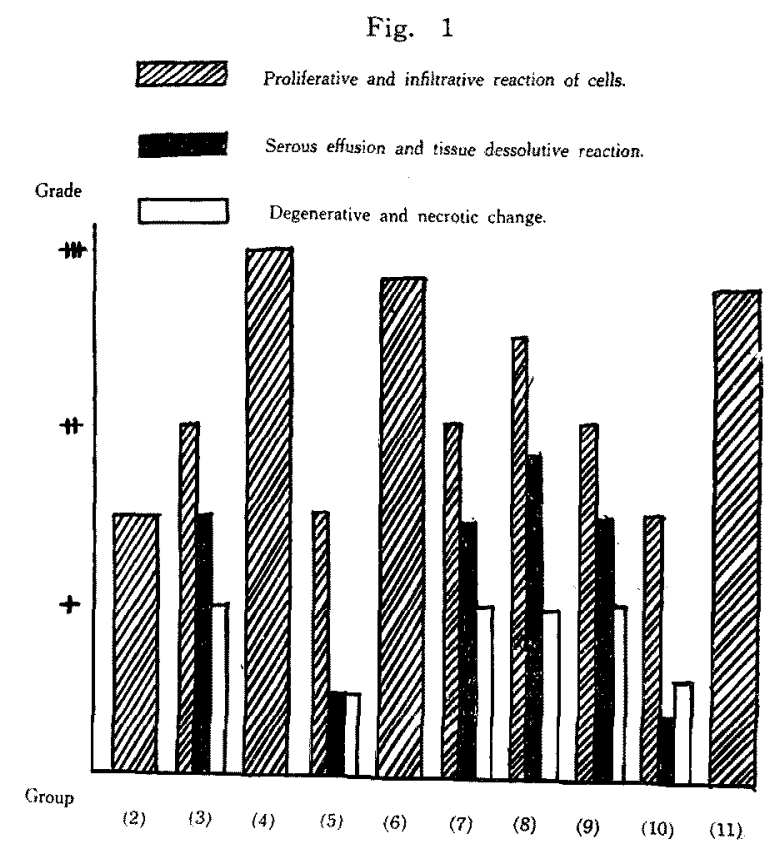


and infiltrative reaction added with the hypofunction (See the Fig. 1).

Many histological studies on sensitized spleen and splenic allergy were already reported(15). Though the findings were various according to the complicity of the splenic tissue and differences of their experimental methods, the results of the present studies were almost similar to them.

Nikoloeff and Goldberg pointed out that when antigen was reinjected in the sensitized animals the injected antigen was intaken and destroyed in the reticuloendothelial system (RES), and that if the antigen destroying power of the RES was weakend or disappeared the regressive degenerations of the vessels and parenchymatous cells were raised. In our experiments, hyperfunction of the RES of the sensitized spleen signified intake and destruction activity caused by reinjection of the antigen, and furthermore, was considered to be the primary cellular reaction accompanied with reaction to the antigen produced in the cells of RES. And appearance of young leukocytes was observed, but as the result of excessive antigen antibody reaction which was moved gradually from hyperfunction to state of exhaustion, and the antigen destroying power diminished. It was considered that, as the secondary tissue reaction, in the RES (also in the lymphocyte system), various grades of destructive degeneration were brought on.

There are some unsettled problems on histochemical demonstration of nucleic acid still now, but our findings, which showed abundance of DNA and RNA, mean synthetic activity of cellular protein and are considered to be the suggestion of production or preservation of them in connection with increase of materials of antigen protein and others ${ }^{(18)}$. In the present observation, the most marked quantity of nucleic acid was demonstrated in lymphogonias and next in reticular cells, lymphoblasts and so on. At the conviction of lymphogonia-Amano, the other kinds of cytochemical knowledges might be necessary as the basis, but the authors noticed some shifting form of the typical reticular cells in the experimental groups of preliminary and later CP treatment and of saline injection, and from their general conditions, the findings of nucleoli, RNA reaction and so on, they presumed provisionally this kind of cells. Amano and others(14) stated that this cell was further younger than the young lymphoblast, and was the lymphocytes, which had been born newly and heterogenenously from reticular cells.

The findings of the groups of preliminary and later treatment with saline solution, performed with contrary meaning, were almost similar to those of the cases of splenic allergy and no characteristic differences were found, but in the cases of preliminary and later CP medication, something, which seemed to be lymphogonia, appeared in a large number, at the time of activation of the RES. 
There was no significant difference between both groups. At any rate, these findings caused by $\mathrm{CP}$ treatment has an extremely great influence upon the splenic allergy. Namely, the findings that at splenic allergy any destructive change was almost not found and striking activity of the RES was seen, revealed the prevention of the exhaustion of all functions of the RES caused by the antigen injection into the spleen, and therefore it can be asserted that $\mathrm{CP}$ restrains powerfully the allergic reaction of the spleen. Then, the reason, that the allergic reaction was restrained in the cases of later CP treatment as well as in the ones of preliminary treatment is easily imaginable to be that the time between the provoking injection and CP medication was an important factor, though in the cases of preliminary $\mathrm{CP}$ treatment the conditions were similar. As in the present experiments the $\mathrm{CP}$ treatment started in comparatively short time after the provoking injection, it was considered that $\mathrm{CP}$ acted intensively before the secondary tissue reaction. Whether $\mathrm{CP}$ acts intensively and constructively even upon the cells, which have already had allergic changes, or not, can not be confirmed, unless a plenty of observations should be performed after various intervals of the procedures.

In the case of simultaneous $\mathrm{CP}$ treatment, unlike the findings of the above two and at the time of splenic allergy, the characteristic finding was atrophy of the RES. Furthermore, in the cases of solitary CP treatment, the findings, similar to those of the cases of simultaneous CP treatment, were found. These findings, when CP acted directly upon the spleen, showed hyperfunction of the RES of the spleen, and explained that the acting mechanism was different from the cases of preliminary and later CP treatment, and all kinds of allergic reactions of the spleen were restrained. In comparison of the groups of instillation of $\mathrm{CP}$ and saline solution, the latter had the findings similar to the groups of preliminary and later CP treatment, and the histological reactions of the both cases were absolutely contrary to each other. Even from these facts, the specific function of CP can be understood. Then, the findings of the group of injection of saline solution were only tissue reaction, which was causually similar, but were raised by the eintirely different mechanism. It was suggested that the findings were results of simple heterogenous reaction, as Reich(19) observed.

It is well known that $C P$ has an extremely specific action upon the central nervous system and restraines powerfully the superior centers of autonomous nerves, or the hypothalamus. On the other hand, morphology has played an important role in investigation of allergic reaction, but about the essential nature there are still now a good deal of theories many controversies. However, Nakamura proved on the basis of numerous fundamental experiments, that 
anaphylaxy was stimulation of the parasympathetic nerves and the stressing point of antigen-antibody reaction was indeed the parasympathetic nerve. The fact that at the cases with allergic reactions the parasympathetic nerve is stimulated and extraordinary hyperfunction of the organs under the control of that nerve is raised, has been also pointed out by Wells. According to him, hyperfunction of sensitized spleen is caused by stimulus of the parasympathetic nerve, and it is considered that the disordered irregularity at the time of splenic allergy suggests the functional confusion of the peripheral branches to the spleen of the parasympathetic nerve, added with excessive stimulation of that nerve.

The theory of Roussy and Mosinger told us that the hypothalamus itself was the correlation place of, not to mention the autonomous nervous system of a living body, but of endocrine system and of all kinds of the other autonomous factors. Considered also from the specific activity of CP upon the hypothalamus, our findings in the group of preliminary and later CP treatment in the present experiment were the result that $C P$ prevented the confusion of the autonomous nervous system and endocrine function, and further in the findings of simultaneous CP medication it was suggested that the anti-autonomous nervous functions of CP acted intensively not only upon the central nerve but upon the peripheries.

While, between the groups of preliminary and later CP treatment and that of simultaneous medication, there were great differences on the point of the appearing phase of the reticuloendothelial and lymphocytic system. What is the reason of this?

Reilly ${ }^{(20)}$ pointed out that, about the connection of the autcnomous nervous and reticuloendothelial system, at the time of normal grade of stimulation of autonomous nerve the function of the RES rised and the excessive stimulation of it brought necrosis. Micklos Jansco told that the activity of the RES of the spleen was restrained by synthesized antihistamic drugs, and, on the other hand, the fact that the center, which controls the RES, lay in the diencephalon, was advocated by Hendel and Malet. Judged from these matters, it is distinct that the findings at the preliminary and later CP treatment expressed the restraint of confusion of the autonomous nervous system, but did not show the complete stopping of the function. Namely, at splenic allergy, excessive stimulation to the splanchnic nerve was restored merely to the state of normal stimulation by CP medication and the state of diencephalonic region seemed to have been still retained. And extraordinary hyperfunction of the RES, as convinced even from the theories of Wells and others, is considered to be the result that, for the sake of intensive antisympathetic nervous function of $\mathrm{CP}$, the antagonistic function of parasympathetic nerve becomes relatively excessive. On the 
other hand, as the influence of $\mathrm{CP}$ to the endocrine system, Laborit and Castaigne pointed out the restraint of function of the pituitoadrenal system. Though this fact is able to be easily convinced without necessary of resembrance of the diencephalon-hypophysis humoral regulation theory, the authors, investigating the spleen of white mice with extirpated adrenal glands, in the connection of histochemical researches of the spleen at the time of secondary shock, noticed the appearance of something like lymphoblasts at the center of follicles and the action of the RES. At the condition in complete disregard of suprarenal function, the destruction of lymphocytes due to stress was not found, and contrarily the splenic function rised. Even compared from the results, it might be able to the understood that the appearance of lymphoblasts and the hyperfunction of RES at the cases of preliminary and later CP treatment in the present experiments, expressed also the intensively restraining action of CP to the pituito-adrenal system.

From these points of view, in the limit of the present experiments, it is considered that CP can almost perfectly intercept mineral corticoid, but its effect to the cholin activating system is rather inferior to that, and although the restraining function is, of course, demonstrable, the stimulating condition is still retained. This problem is, however, not easily concluded, because the function of CP may be changeable, especially according to the quantitative difference and the various kinds of mysterious reactions of living organism to stress are not yet made clear.

In the present experiments, the dose of $\mathrm{CP}$ in the cases of preliminary treatment was $0.2 \mathrm{mg} \times 5 / \mathrm{kg}$ which was rather small as a whole body dose in animals. In the cases of preliminary and later treatments, CP was injected into the auricular vein for the whole body, and compared with the cases of simultaneous treatment, its activation of the superior center was further intensive and, on the contrary, the quantity to the spleen itself was considered to be extremely small. On the other hand, it can be promised that in the cases of simultaneous treatment, CP acted directly and the quantity was large. The result that for the former the splenic function rised extraordinaly, and at the latter the function declined somewhat remarkably, was obtained.

The mechanism of $\mathrm{CP}$ function upon the tissue cells might be different, whether to whole body or to locals, but to discuss the changes due to the quantitative differences of cytological reaction depend on the medicating methods of $\mathrm{CP}$, the period of observation and other factors. Decourt(22) pointed out that CP decreased the cell functions, but judged from the present results, that matter could be asserted only when CP acted directly to the cells, and when CP 
functioned to whole body, the result was contrary. Then, it is considered that the problem could not simply concluded.

As above mentioned, on the histological points of view, the authors conclude that Chlorpromazine has remarkably restraining effects to the experimental splenic tissue allergy, with some discussions.

\section{CONCLUSION}

1. After preliminary, simultaneous and later injection of Chlorpromazine $0.2 \mathrm{mg} \times 5 / \mathrm{kg}$ into the auricular vein of rabbits, experimental tissue allergy of the spleen was inhibited in all cases.

2. In the cases of injection of Chlorpromazine $0.2 \mathrm{mg}$ to the splenic artery and of the antigen (horse serum $0.2 \mathrm{ml}$ ), allergic response was also inhibited.

3. After sensitation, the treatment by CP to whole body activated the RES of the spleen with the production of young lymphocytes, and the local medication of CP provoked atrophy of the RES in the spleen with degenerative leukocytes.

4. The antipathic function of Chlorpromazine to the autonomous nervous system was considered to be effective even in peripheries. And the drug inhibited intensively the activity of mineral corticoid with the cholin activating center. Moreover, to understand the grades of the functions, further spread investigations, concerning to the influences according to the quantitative differences of Chlorpromazine, must be necessary.

\section{REFERENCES}

1. Courvoiser, S.; Fournel, J.; Ducrot, R. et Kolsky, M.: Arch. int. pharmacodyn., 92: 305, 1953.

2. Donnet, V.; Zwirn, P. et Ardison, J. L.: C. R. S. B.: 148: 1617, 1953.

3. Shibusawa, K.: Saishin Igaku, 10: 2393, 1955 (in Japanese).

4. Kobayashi, T.: Nichifutsu Igaku, 2: 2, 1955 (in Japanese).

5. Kobayashi, T.: Saishin Igaku, 11: 462, 1956 (in Japanese).

6. Nakayama, K.: Jap. J. of Allergy, 5: 75, 1956 and Keio J. of Med., 6: 1, 1957.

7. Lillie, A. B.: Histopathologic Technic, New York, 1953.

8. Steadman, E.: Symp. Soc. Exp. Biol., 1: 232, 1953.

9. Alfert, A. M.: J. Cell. \& comp. physiol., 36:381, 1950.

10. Stowell, R. F. \& Albers, V. M.: Stain Technol., 18: 57, 1943.

11. Yajima, G. et al.: Transact. Soc. Path. Jap., 43: 575, 1954.

12. Amano, S. et al.: Jap. J. of Hemat., 14: 58, 1951 (in Japanese).

13. Amano, S. et al.: Acta Path. Jap., 1: 117, 1951.

14. Umino, G.: Jap. J. of Hemat., 16: 10, 1953 (in Japanese).

15. Kirihara, Y.: not published. 
16. Nakadate, K.: Jap. Med. J., 1665: 1956 (in Japanese) .

17. Okabayashi, A.: Saishin Igaku, 8, 7, 750, 1953 (in Japanese).

18. Caspersson, T.: Skand. A. Phisiol. Supp. Nr. 8. zum Bd. 73, 1936.

19. Rich, A. R. et al.: Bull. Johns. Hopk. Hosp., 65: 271, 311, 1939.

20. Reilly, G.: C. R. Soc. Bio., 116: 24, 1934.

21. Isono, N.: not published.

22. Decourt, P.: Presse Med., 62: 541, 1954. 


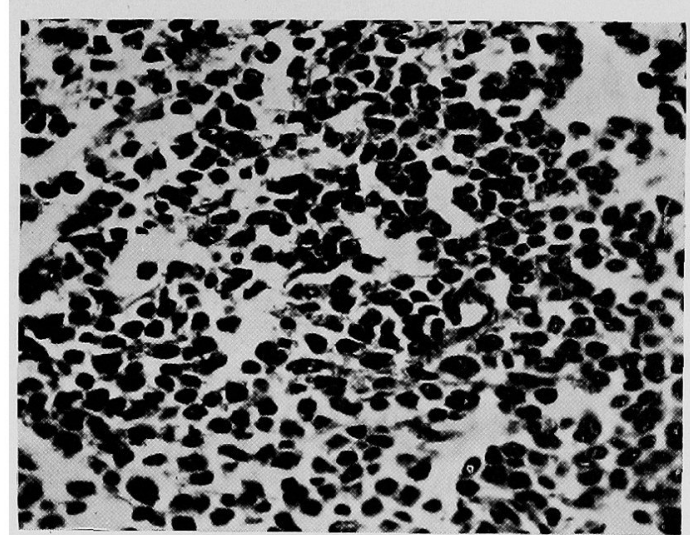

Fig. 2 Surrounding regions of germinal center of lymphfollicles of spleen (Control).

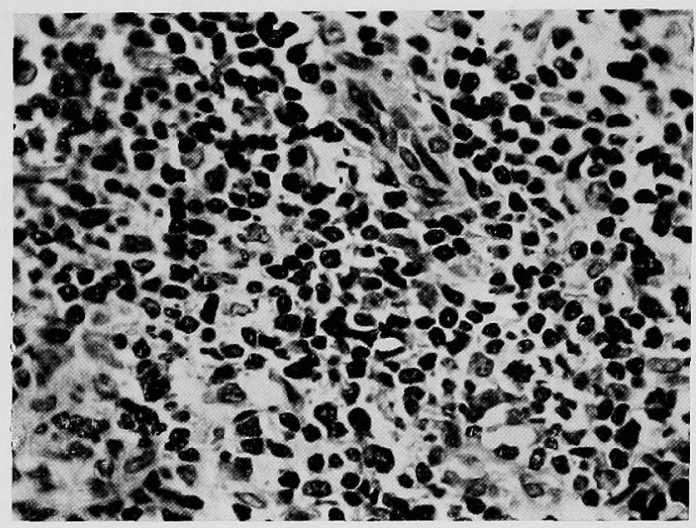

Fig. 3 Case of sensitation.

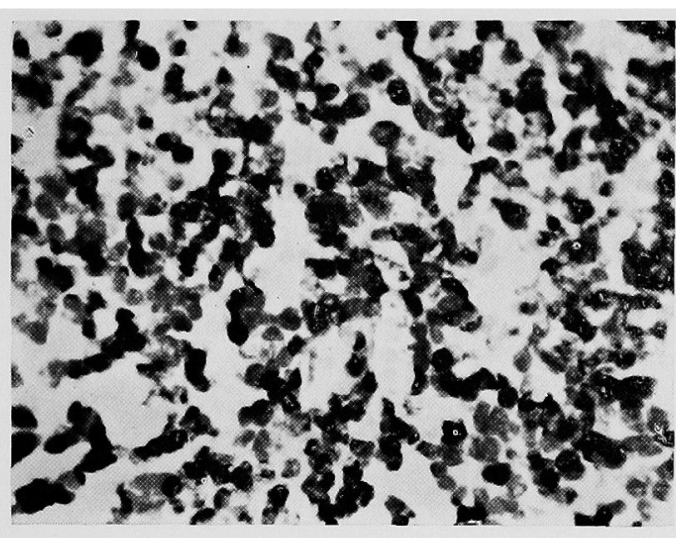

Fig. 4 Case of tissue allergy.

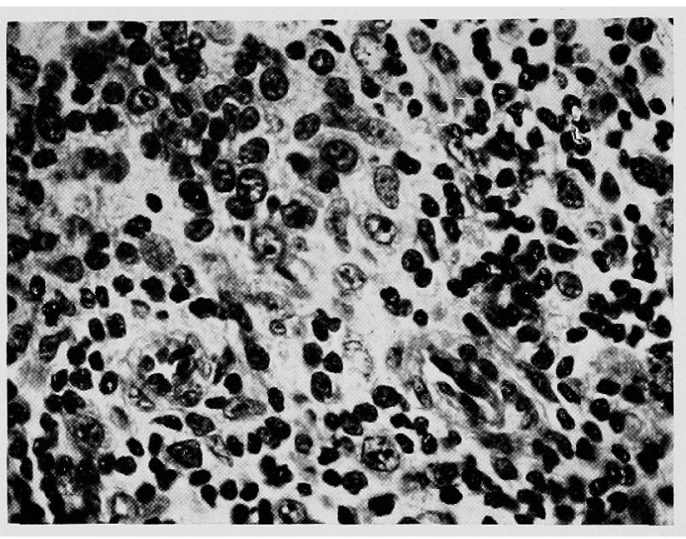

Fig. 5 Case of preliminary $\mathrm{CP}$ treatment.

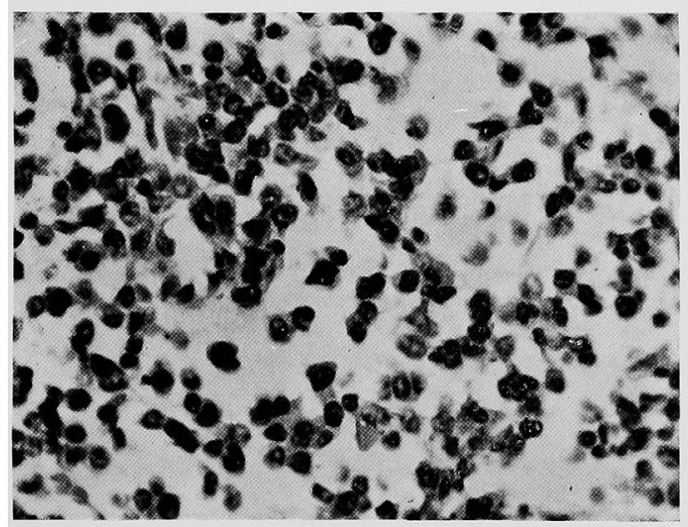

Fig. 6 Cace of simultaneous CP treatment.

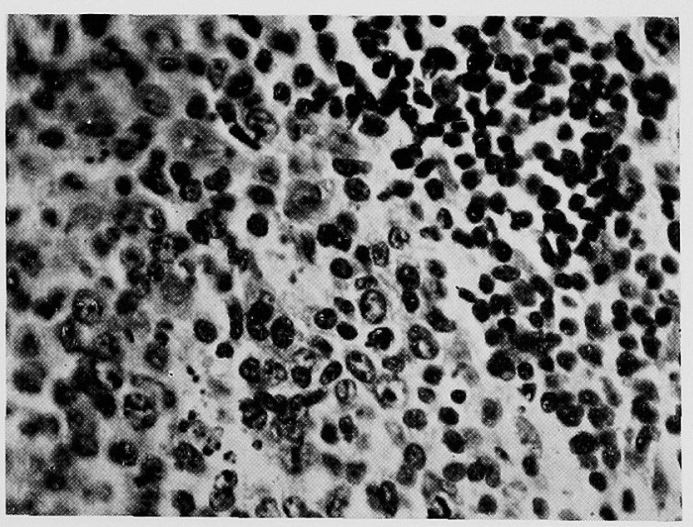

Fig. 7 Case of later $\mathrm{CP}$ treatment. 


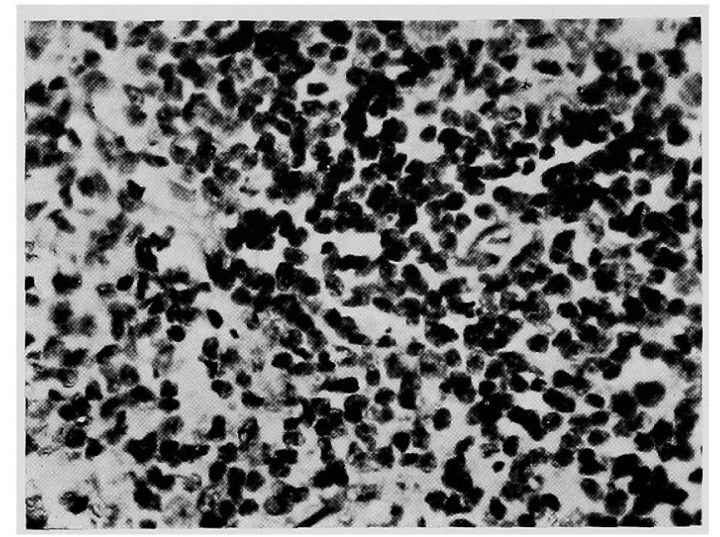

Fig. 8 Case of preliminary treatment with saline solution.

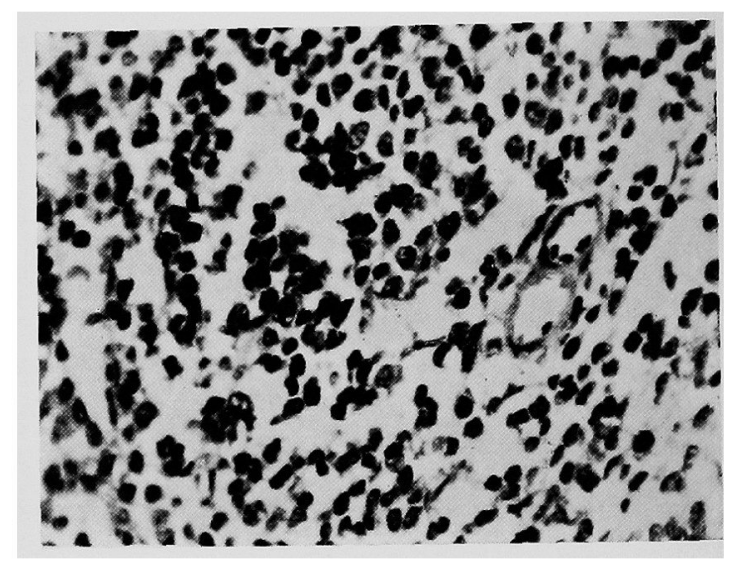

Fig. 9 Case of $\mathrm{CP}$ injection.

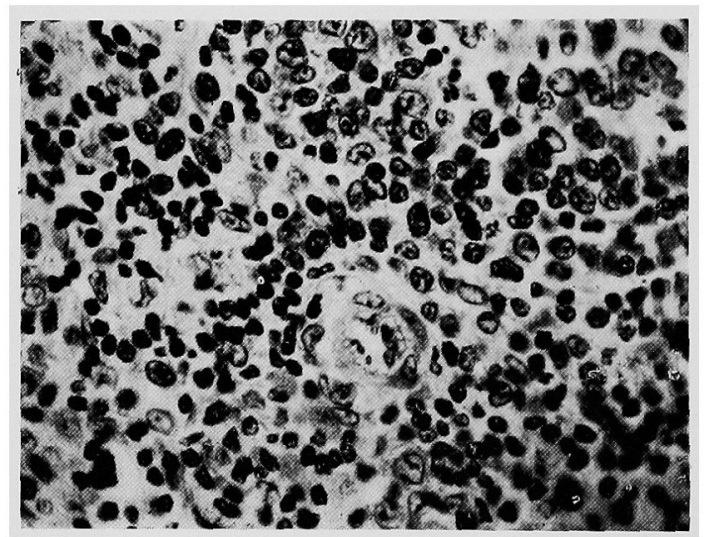

Fig. 10 Case of saline injection. 